\title{
Genes encoding histone proteins are differentially expressed between men and women with diffuse intrinsic pontine glioma and their expression correlates with survival.
}

\author{
Shahan Mamoor ${ }^{1}$ \\ 1Thomas Jefferson School of Law \\ mamoorsk@tisl.edu \\ San Diego, CA 92901
}

Diffuse intrinsic pontine glioma is a pediatric brain cancer and has the lowest median survival rate of all cancers known to man (1). 99\% of patients diagnosed with DIPG will expire within 5 years (1). Understanding the transcriptional behavior of tumors in DIPG is critical for the development of novel therapies. In this study, I compared the transcriptomes of tumors from men with DIPG versus that of tumors from women diagnosed with DIPG using a published dataset (2). I found that three histone genes, including HIST1H4C, HIST1H2BD, and HIST1H3D, which encode Histone H4, Histone H2B Type 1D, and Histone H3.1 were among the genes whose expression was most different between the DIPG tumors of men and women. Importantly, the expression level of two of these genes significantly correlated in a linear fashion with the amount of time the patient survived. It has previously been reported that $78 \%$ of DIPG tumors contain a mutation in Histone H3.1 (HIST1H3B) (3). This is the first report of differential expression of histone genes in tumors of patients with DIPG.

Keywords: diffuse intrinsic pontine glioma, DIPG, systems oncology, tumor transcriptome, differential gene expression analysis, histone alterations in cancer, H3.1, H2B Type 1D, H4, 


\section{Introduction}

Diffuse intrinsic pontine glioma is a pediatric brain cancer with the worst prognosis of all cancers - the 5 year median survival rate is $99 \%$. To more thoroughly understand the major transcriptional differences between males and females diagnosed with this cancer, we performed global differential gene expression analysis, using a dataset to compare the transcriptomes of the tumors of males and the tumors of females. We found that genes encoding 3 separate histone proteins were among the most differentially expressed when comparing tumors by the sex of the patient. Significantly, the expression of two of these histone genes displayed linear and significant correlation with the amount of time the patient survived. These results merit further study into the roles of these histone subunits in the biology of DIPG.

\section{Methods}

Dataset GSE50021 (2) was utilized for this analysis, in conjunction with GEO2R, to perform differential gene expression analysis on tumor samples from patients diagnosed with DIPG ( $n=35 ; n=19$ males and $n=16$ females) . Buczkowicz et al. (2) used Illumina HT-12 microarray analysis to obtain transcriptome data from DIPG tumor samples. These samples were all fresh frozen paraffin embedded tumors from pediatric patients. The Benjamini and Hochberg method of $p$-value adjustment was used. Log transformation was set to "Autodetect" and the submitter supplied category of platform annotation was used. For statistical analysis to compare the RNA expression values between male and female tumor groups, an unpaired, two-tailed t-test with Welch's correction was used (PRISM 8.1.2) (227). Linear regression analysis and a Pearson correlation, comparing the mRNA expression level of each patient and the time amount of time they survived, were also performed using PRISM. 


\section{Results}

We compared the transcriptomes of tumors from males with the transcriptomes of tumors from females in patients diagnosed with diffuse intrinsic pontine glioma (DIPG). We found that of the most significantly differentially expressed genes in the DIPG transcriptome when comparing sex of the tumor, 3 were genes encoding histone proteins. These included HIST1H4C which encodes Histone H4, HIST1H2BD which encodes Histone H2B Type 1D, and HIST1H3D which encodes Histone H3.1.

Genes encoding histone proteins are differentially expressed when comparing the transcriptomes of tumors from men and women with DIPG.

HIST1H4C, the gene for Histone H4, was the 83rd most differentially expressed gene out of 29285 total transcripts detected by the microarray data utilized in this analysis, when comparing the tumors of males and the tumor of females (Table $1 ; p=4.2 \mathrm{E}-03$ ). HIST1H2BD, the gene for Histone H2B Type 1D, was the 175th most differentially expressed gene out of 29285 total transcripts detected by the microarray data utilized in this analysis, when comparing the tumors of males and the tumor of females (Table $1 ; p=1.12 \mathrm{E}-02$ ). The third differentially expression histone gene, HIST1H3D, the gene for Histone H3.1, was the 212nd most differentially expressed gene out of 29285 total transcripts detected by the microarray data utilized in this analysis, when comparing the tumors of males and the tumor of females (Table $1 ; p=1.4 \mathrm{E}-02)$.

HIST1H4C, HIST1H2BD and HIST1H3D are among the genes most differentially expressed between the tumors of men and women with diffuse intrinsic pontine glioma, and expressed at significantly higher levels in males.

Next, we extracted the individual mRNA expression values for each histone gene, in every patient tumor ( $n=19$ males, $n=16$ females). HIST1H4C was expressed at significantly higher levels in the tumors of males compared to the tumors of females (Figure $1 ; p=0.0120$ ). HIST1H2BD was expressed at significantly higher levels in the tumors of males compared to 
the tumors of females. (Figure $2 ; p=0.0073$ ). HIST1H3D was also expressed at significantly higher levels in the tumors of males compared the tumors of females (Figure $3 ; p=0.0183$ ).

Expression of histone genes HIST1H4C and HIST1H2BD in DIPG tumors display significant and linear correlation with the amount of time the patient will survive.

Finally, we evaluated if the expression of any of these genes correlated with patient outcomes. HIST1H4C expression at the mRNA level displayed a significant correlation with patient overall survival when linear regression analysis was performed, comparing the mRNA expression level of each patient with the amount of time the patient survived (Figure 4; $p=0.0022)$. HIST1H2BD (Figure $4 ; p=0.0071$ ) mRNA expression also displayed a significant correlation with patient overall survival when a linear regression analysis was performed, comparing the amount of HIST1H2BD that was expressed with the amount of time the patient survived (Figure 4: 0.0071). HIST1H3D, at the mRNA level, did not display a significant correlation with patient overall survival.

\section{Discussion}

Diffuse intrinsic pontine glioma (DIPG) has the worst patient outcomes of any cancer. To understand the transcriptional behavior of FIPG tumors as it differs between men and women with the disease, we performed global differential gene expression analysis, comparing the transcriptomes of tumors from men versus the transcriptomes of tumors from women. We found that three separate histone genes were differentially expressed between men and women with DIPG. These included HIST1H4C, HIST1H2BD, and HIST1H3D.

There is a lack of published information available regarding $\mathrm{HIST} 1 \mathrm{H} 4 \mathrm{C}$ and cancer. HIST1H2BD has been reported as part of a gene-signature that predicts prognosis in cervical cancer (4). However, a study that sequenced the tumors of patients with DIPG found that the gene encoding Histone H3.1, HIST1H3BD, is mutated in $78 \%$ of diffuse intrinsic pontine gliomas (3). Interestingly, another group found that this mutation in Histone H3.1 lead to decreased EZH2 activity at position 27 (otherwise a lysine and not a methionine), and 
subsequent decreased methylation at that position of the chromatin. The methylation mark at H3K27 is normally otherwise a silencing mark and thus this mutation, found in DIPG tumors, lead to loss of a silencing epigenetic mark (5).

In gastric cancer, HIST1H3D expression is higher in the peripheral blood, bone marrow and primary tumor of stage IV gastric cancer patients than in the peripheral blood, bone marrow and primary tumor of stage I gastric cancer patients (6).

In pediatric glioblastoma, Histone H3.3 (encoded by H3FA) is mutated in nearly $33 \%$ of cases at position G34 (7).

These previously published data indicate that the genes encoding histones are mutated in cancers, including specifically in diffuse intrinsic pontine glioma, and our data here demonstrate that three histone genes are differentially expressed when comparing the tumor transcriptomes from men with that of women, that each of these three genes are expressed at higher levels in the tumors of men than in women, and that two of these genes display a significant and linear correlation with the amount of time the patient will survive. Together, these data indicate that histones are an important biological factor to consider in the etiology, prognosis, and potentially the treatment of the deadliest cancer known to man, diffuse intrinsic pontine glioma. 


\section{References}

1. Vanan, M.I. and Eisenstat, D.D., 2015. DIPG in children-what can we learn from the past?. Frontiers in oncology, 5, p.237.

2. Buczkowicz, P., Hoeman, C., Rakopoulos, P., Pajovic, S., Letourneau, L., Dzamba, M., Morrison, A., Lewis, P., Bouffet, E., Bartels, U. and Zuccaro, J., 2014. Genomic analysis of diffuse intrinsic pontine gliomas identifies three molecular subgroups and recurrent activating ACVR1 mutations. Nature genetics, 46(5), p.451.

3. Wu, G., Broniscer, A., McEachron, T.A., Lu, C., Paugh, B.S., Becksfort, J., Qu, C., Ding, L., Huether, R., Parker, M. and Zhang, J., 2012. Somatic histone H3 alterations in pediatric diffuse intrinsic pontine gliomas and non-brainstem glioblastomas. Nature genetics, 44(3), p.251.

4. Li, X., Tian, R., Gao, H., Yang, Y., Williams, B.R., Gantier, M.P., McMillan, N.A., Xu, D. and $\mathrm{Hu}, \mathrm{Y} ., 2017$. Identification of a histone family gene signature for predicting the prognosis of cervical cancer patients. Scientific reports, 7(1), p.16495.

5. Lewis, P.W., Müller, M.M., Koletsky, M.S., Cordero, F., Lin, S., Banaszynski, L.A., Garcia, B.A., Muir, T.W., Becher, O.J. and Allis, C.D., 2013. Inhibition of PRC2 activity by a gain-offunction H3 mutation found in pediatric glioblastoma. Science, 340(6134), pp.857-861.

6. Iwaya, T., Fukagawa, T., Suzuki, Y., Takahashi, Y., Sawada, G., Ishibashi, M., Kurashige, J., Sudo, T., Tanaka, F., Shibata, K. and Endo, F., 2013. Contrasting expression patterns of histone mRNA and microRNA 760 in patients with gastric cancer. Clinical Cancer Research, 19(23), pp.6438-6449.

7. Schwartzentruber, J., Korshunov, A., Liu, X.Y., Jones, D.T., Pfaff, E., Jacob, K., Sturm, D., Fontebasso, A.M., Quang, D.A.K., Tönjes, M. and Hovestadt, V., 2012. Driver mutations in histone H3. 3 and chromatin remodelling genes in paediatric glioblastoma. Nature, 482(7384), p.226. 


\section{HIST1H4C}

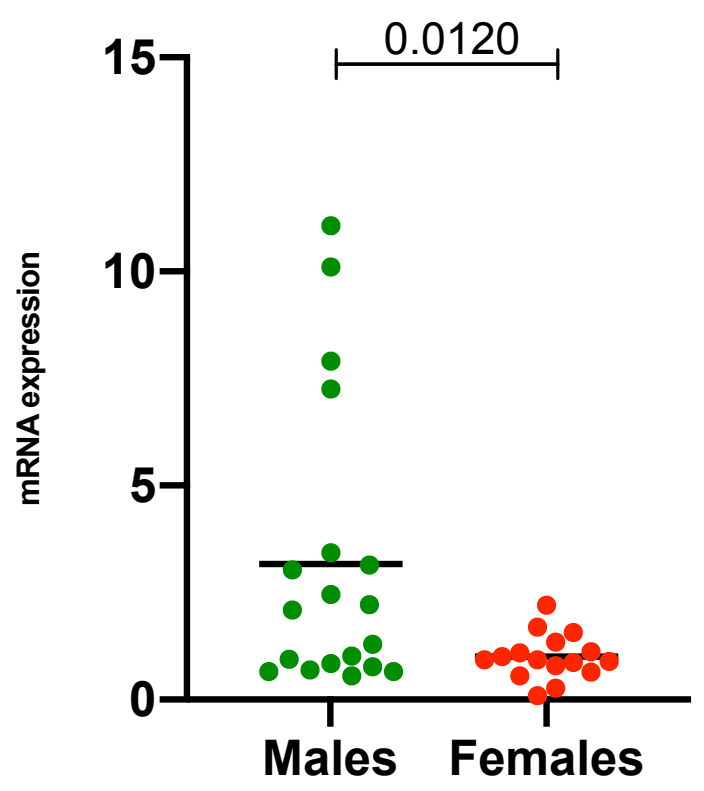

\section{HIST1H4C}

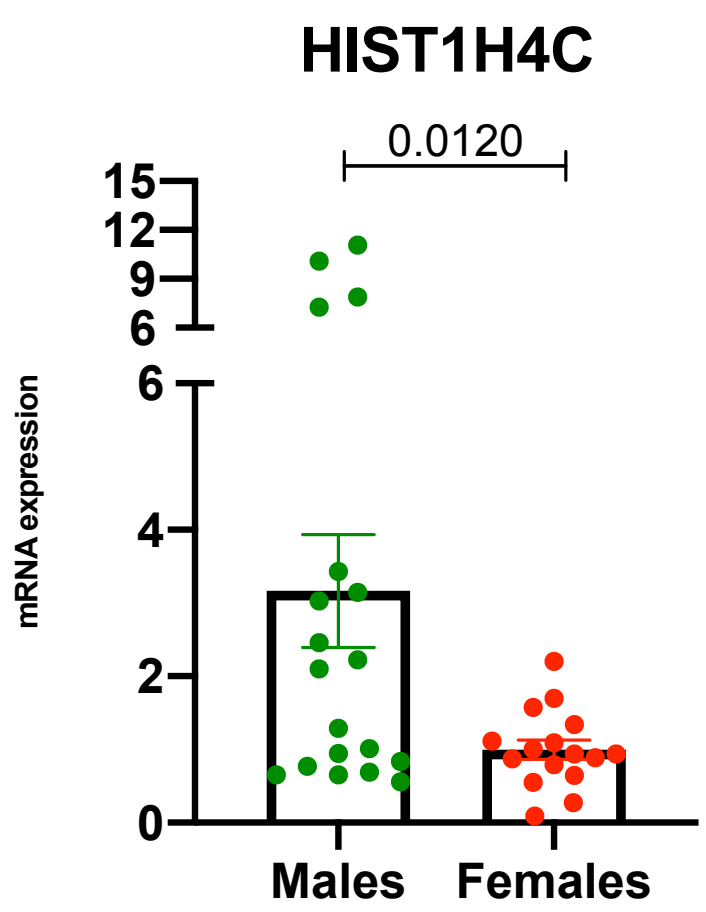

Figure 1: HIST1H4C is among the genes most differentially expressed between the tumors of men and women with diffuse intrinsic pontine glioma, and expressed at significantly higher levels in males.

The expression level of HIST1H4C in men and women is graphically represented, with one dot representing the expression level the histone gene in one individual patient's tumor. The horizontal black bar represents the mean expression in each sex. A statistical test was performed to evaluate the significance of the difference in expression level between each sex, and the result is shown as a $p$-value on each graph. Below is the same data but in bar graph format, with the error bars representing the standard error of the mean. 


\section{HIST1H2BD}

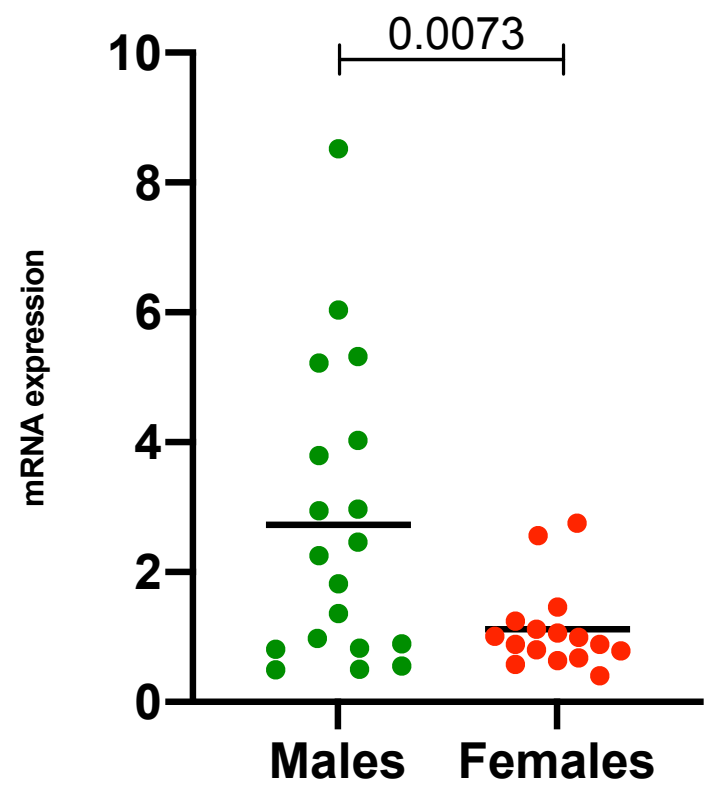

\section{HIST1H2BD}

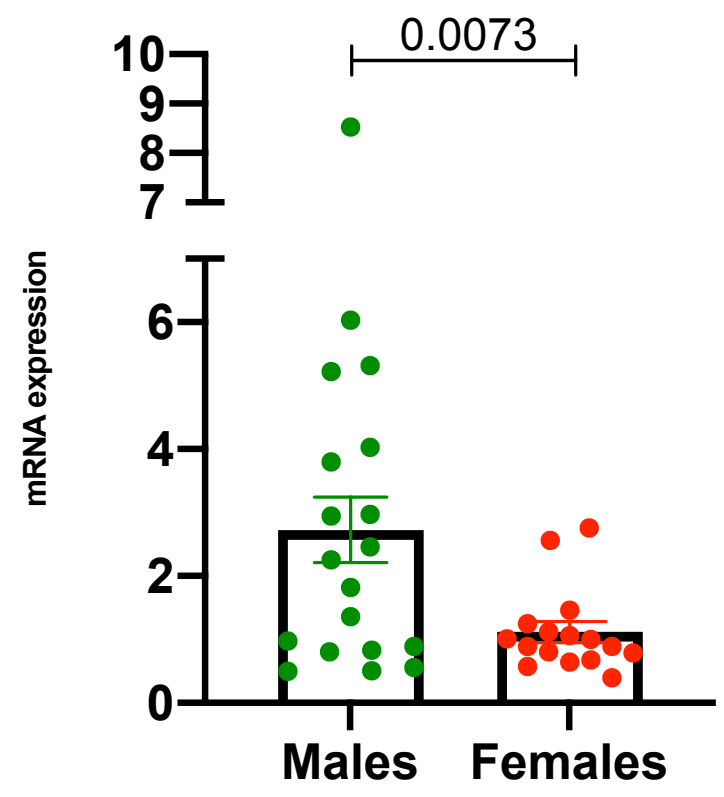

Figure 2: HIST1H2BD is among the genes most differentially expressed between the tumors of men and women with diffuse intrinsic pontine glioma, and expressed at significantly higher levels in males.

The expression level of HIST1H2BD in men and women is graphically represented, with one dot representing the expression level the histone gene in one individual patient's tumor. The horizontal black bar represents the mean expression in each sex. A statistical test was performed to evaluate the significance of the difference in expression level between each sex, and the result is shown as a $p$-value on each graph. Below is the same data but in bar graph format, with the error bars representing the standard error of the mean. 


\section{HIST1H3D}

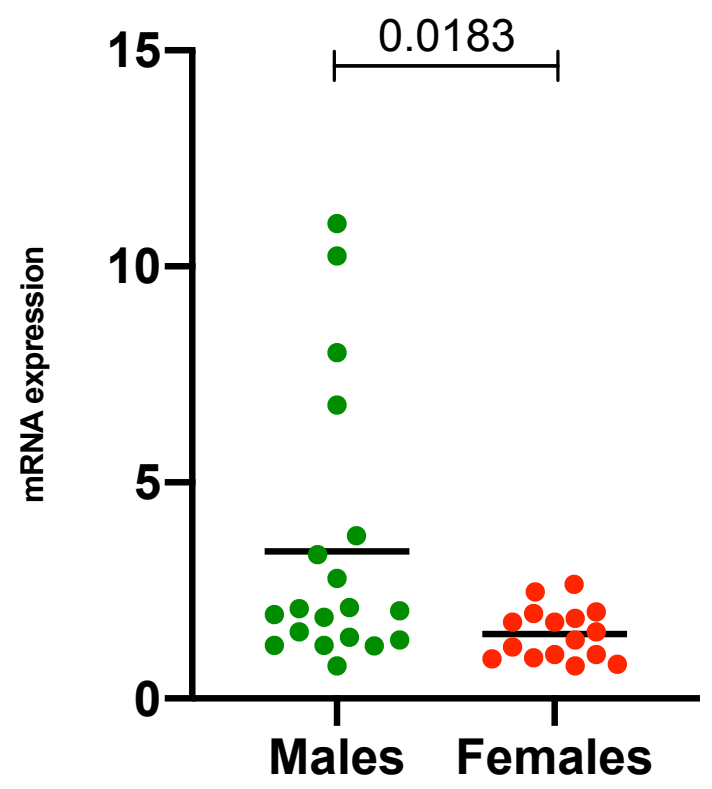

\section{HIST1H3D}

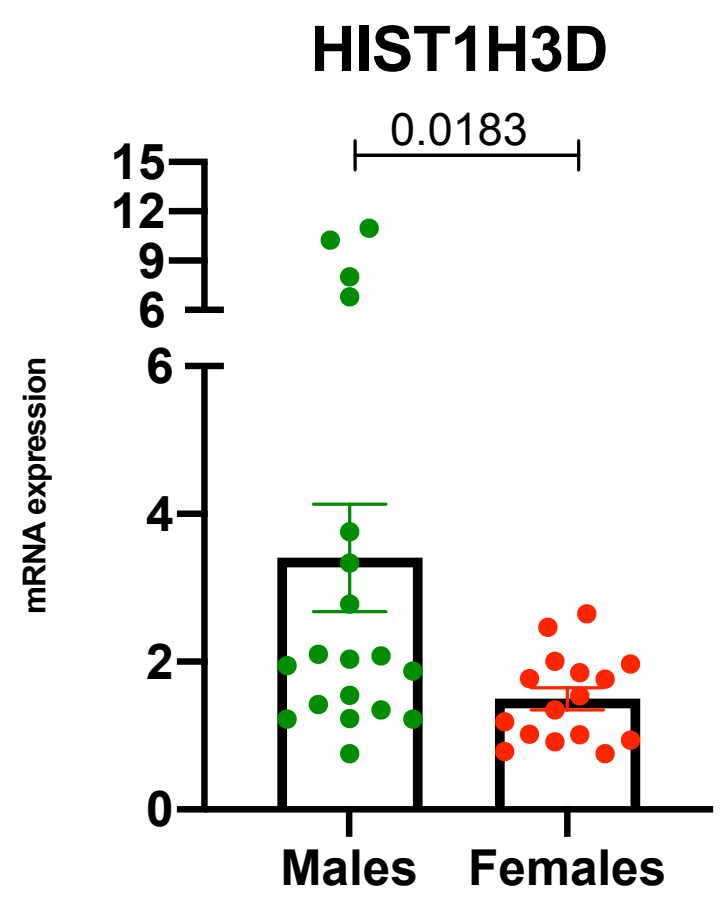

Figure 3: HIST1H3D is among the genes most differentially expressed between the tumors of men and women with diffuse intrinsic pontine glioma, and expressed at significantly higher levels in males.

The expression level of HIST1H3D in men and women is graphically represented, with one dot representing the expression level the histone gene in one individual patient's tumor. The horizontal black bar represents the mean expression in each sex. A statistical test was performed to evaluate the significance of the difference in expression level between each sex, and the result is shown as a $p$-value on each graph. Below is the same data but in bar graph format, with the error bars representing the standard error of the mean. 

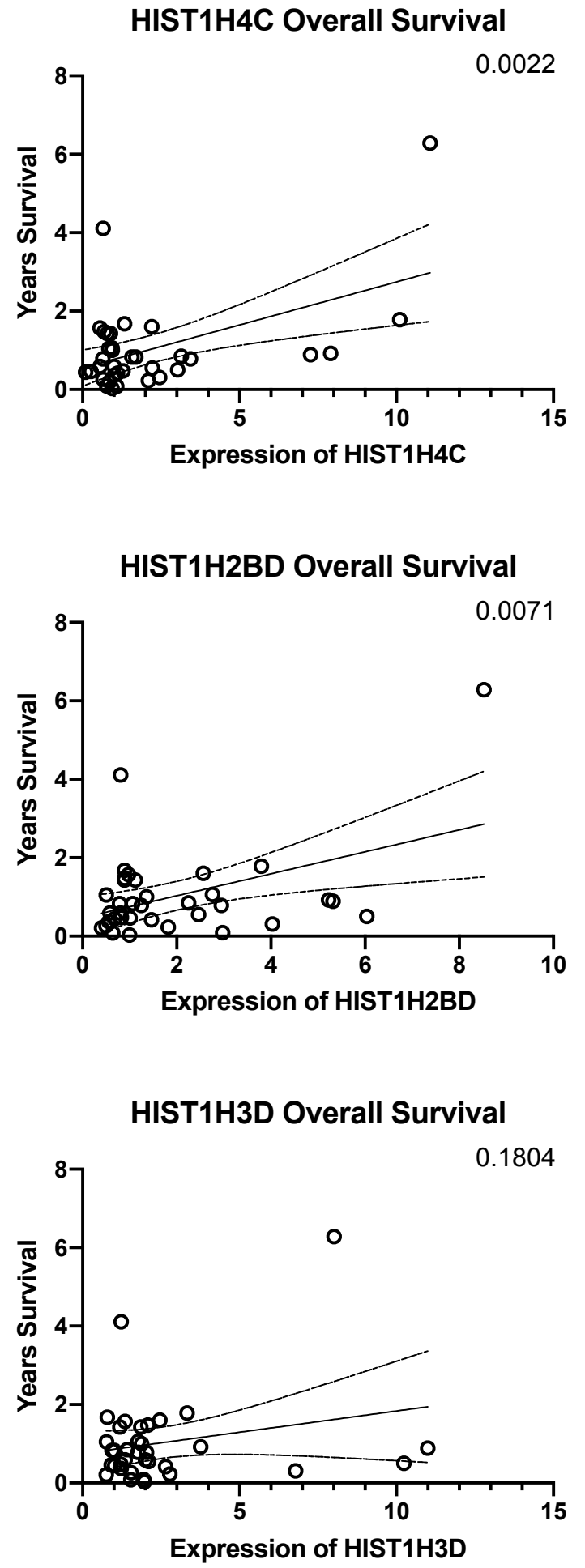

Figure 4: Expression of histone genes HIST1H4C and HIST1H2BD in DIPG tumors display significant and linear correlation with the amount of time the patient will survive.

The expression of each differentially expressed histone gene in the brain tumors of patients with DIPG was plotted against the amount of time the patient survived (overall survival). A statistical test was performed to assess the linearity and non-zero quality of the correlation, and is shown as a $p$-value on each graph. 
Table 1

\begin{tabular}{|c|c|c|c|c|c|c|c|}
\hline Rank & Symbol & $p$-value & $\mathbf{t}$ & B & ID & Chr. & GB_ACC \\
\hline 83 & HIST1H4C & $4.2 \mathrm{E}-03$ & -3.0022663 & -2.5436 & ILMN_2075334 & 6 & NM_003542.3 \\
\hline 175 & HIST1H2BD & 1.12E-02 & -2.6350457 & -3.0751 & ILMN_1758623 & 6 & NM_138720.1 \\
\hline 212 & HIST1H3D & $1.4 \mathrm{E}-02$ & -2.548034 & -3.1946 & ILMN_1721127 & 6 & NM_003530.3 \\
\hline
\end{tabular}

Table 1: Genes encoding histone proteins are differentially expressed when comparing the transcriptomes of tumors from men and women with DIPG.

The rank of expression out of 29285 total transcripts, the gene symbol, the $p$-value of differential expression globally, a moderated $t$-statistic $(\mathrm{t}), \mathrm{B}$, the log-odds of differential expression between both groups and the GenBank accession ID are displayed in this table (all provided by GEO2R). 